\title{
Uso do laser de baixa potência no tratamento de lesões bucais em pacientes com doenças neurológicas: Relato de caso
}

Use of low power laser in the treatment of oral injuries in patients with neurological diseases: Case report

Uso del láser de baja potencia en el tratamiento de lesiones orales en pacientes con enfermedades neurológicas: Reporte de caso

Recebido: 14/05/2021 | Revisado: 20/05/2021 | Aceito: 21/05/2021 | Publicado: 07/06/2021

Brenna Fernanda Melo Barros ORCID: https://orcid.org/0000-0003-1960-6531 Universidade Federal do Maranhão, Brasil E-mail: brenna.fmelo@gmail.com Cayara Mattos Costa ORCID: https://orcid.org/0000-0002-4491-9700 Universidade Federal do Maranhão, Brasil E-mail: cayara_mattos@hotmail.com

Graça Maria Lopes Mattos ORCID: https://orcid.org/0000-0003-2460-0169 Universidade Ceuma, Brasil E-mail:gm.mattos@hotmail.com

Natália de Castro Côrrea

ORCID: https://orcid.org/0000-0003-1121-7953 Prefeitura Municipal de Chapadinha, Brasil E-mail:natcorra6@gmail.com

Rosana Costa Casanovas

ORCID: https://orcid.org/0000-0002-6871-3491 Universidade Federal do Maranhão, Brasil E-mail: rosana.c.casanovas@hotmail.com

\section{Resumo}

A Terapia de Fotobiomodulação com o Laser de Baixa Potência tem sido utilizada tanto na prevenção quanto no tratamento de lesões bucais. Objetivo: relatar um caso sobre o tratamento de lesão bucal traumática com a terapia de fotobiomodulação. Metodologia: Partindo da permissão por meio do Termo de Consentimento Livre e Esclarecido (TCLE) em partilhar as imagens para devido fim, o presente estudo trata de um relato de caso de cunho analítico descritivo. Relato de caso: Paciente do sexo masculino, 43 anos, proveniente de hospital em São Luís, foi diagnosticado com novo Acidente Vascular Cerebral isquêmico (AVCi). Durante internação evoluiu de tubo orotraqueal para traqueostomia, realizando autofagia do lábio inferior como consequência da falta de coordenação dos movimentos musculares e potencializados durante manipulação. Ao exame físico odontológico, os dentes 11 e 12 estavam vestibularizados, com lesão em lábio inferior extensa; foi utilizada a cânula de Guedel e prescrito Triancinolona Acetonida como terapia. Após avaliação odontológica, o plano terapêutico constituiu-se de remoção imediata de cânula, otimização da analgesia, confecção de placa oclusal, desgaste de faces incisais de dentes 11 e 12 , suspensão da Triancinolona, higienização da área e aplicação de laser de baixa potência com comprimento de onda de $660 \mathrm{~nm}$, potência de $100 \mathrm{~mW}$, tempo 20 segundos, energia 2J, em 3 sessões. Resultado: Observou-se a regressão da lesão após 24 horas da aplicação do laser. Manteve-se o uso de placa e acompanhamento após alta da Unidade de Terapia Intensiva. Conclusão: $O$ uso da terapia de fotobiomodulação mostrou-se uma ferramenta eficaz na cicatrização de lesão bucal traumática.

Palavras-chave: Lasers; Úlceras orais; Autofagia.

\begin{abstract}
The therapy of Photobiomodulation with Low Power Laser has been used in the prevention and handling of oral injuries. Objective: to report a case on the treatment of traumatic oral injury with photobiomodulation therapy. Methodology: Based on the permission through the Free and Informed Consent Term (ICF) to share the images for due purpose, the present study deals with a case report of a descriptive analytical nature. Case report: A 43-year-old male patient from a hospital in São Luís was diagnosticated with a new ischemic stroke. Over the hospitalization after dependence on mechanical ventilation, the patient developed from an orotracheal tube to a tracheostomy, exponent autophagy of the lower lip as a consequence foul of muscular coordination and potentialized movements during manipulation. On physical odontological exam, the teeth 11 and 12 were, with huge injury on lower lip; using the
\end{abstract}


Guedel cannula and where Triamcinolone Acetonide was prescribe as therapy. After the dental appreciation, the treatment plan consisted in removing instantly the cannula, optimization of analgesia, fabrication of occlusal plaque, wear of incisal faces of teeth 11 and 12, hanging of Triamcinolone,clean the area and application of low-power laser with the wavelength $660 \mathrm{~nm}, 100 \mathrm{~mW}$ of power, time of 20 seconds, 2J of energy, in 3 sessions. Result: Was observed in 24 hours after the laser application the injury regression. were maintained the use of plaque and follow-up after discharge from the ICU. Conclusion: The use of the photobiomodulation therapy proved to be an effective tool in the healing of traumatic oral injuries.

Keywords: Lasers; Oral ulcers; Autophagy.

\section{Resumen}

La terapia de fotobiomodulación con láser de baja potencia se ha utilizado tanto en la prevención como en el tratamiento de lesiones bucales. Objetivo: reportar un caso sobre el tratamiento de lesión oral traumática con terapia de fotobiomodulación. Metodología: Partiendo del permiso a través del Formulario de Consentimiento Libre e Informado (CIF) para compartir las imágenes con la debida finalidad, el presente estudio trata de un caso clínico de carácter analítico descriptivo. Caso clínico: Un paciente masculino de 43 años de un hospital de São Luís fue diagnosticado de un nuevo Accidente Cerebrovascular isquémico. Durante la hospitalización, el paciente evolucionó de un tubo orotraqueal a una traqueotomía, realizándose autofagia del labio inferior como consecuencia de la falta de coordinación de los músculos y potencializados durante la manipulación. Al examen físico dental, los dientes 11 y 12 estaban vestibularizados, con lesión en el labio inferior extenso; se utilizó la cánula de Guedel y se prescribió acetónido de triamcinolona como terapia. Luego de una evaluación dental, el plan terapéutico consistió en remoción inmediata de cánula, optimización de analgesia, fabricación de placa oclusal, desgaste de incisales de dientes 11 y 12 , suspensión de Triamcinolona, limpieza de la zona y aplicación de láser de baja potencia con longitud de onda $660 \mathrm{~nm}$, potencia $100 \mathrm{~mW}$, tiempo 20 segundos, energía 2J, en 3 sesiones. Resultado: Se observó regresión de la lesión 24 horas después de la aplicación del láser. El uso de placa y el seguimiento continuó después del alta de la Unidad de Cuidados Intensivos. Conclusión: El uso de la terapia de fotobiomodulación demostró ser una herramienta eficaz en la curación de lesiones orales traumáticas.

Palabras clave: Rayos láser; Úlceras bucales; Autofagia.

\section{Introdução}

O Acidente Vascular Cerebral (AVC) é caracterizado como um déficit neurológico focal ou global, sendo classificado como isquêmico quando ocorre a obstrução de um ou mais vasos, ou hemorrágico, quando há a ruptura do vaso, causando hemorragia em alguma parte do cérebro (Santos \& Waters, 2020). Acomete principalmente idosos, quase três quartos dos derrames acontecem em pessoas com mais de 65 anos. Hipertensão arterial sistêmica (HAS), diabetes mellitus (DM) e tabagismo são os principais fatores de risco. Apresenta danos nas funções neuropsicológicas, dando origem a deficiências motoras, alteração de tônus muscular, sensoriais, de comportamento e linguagem, podendo ocasionar sequelas como disfagia, comprometimento de movimentos e deficiência na força mastigatória e força dos lábios, tendo potencial de ocasionar lesões orais pela falta de coordenação dos movimentos musculares (Gomes, 2019; Santos \& Waters, 2020)

Diante de uma doença grave e limitante, visando oferecer dignidade e diminuição do sofrimento desses pacientes, pelo nível de comprometimento e início súbito de sintomas, justifica-se a necessidade de uma abordagem voltada aos cuidados paliativos dentro da equipe multiprofissional (Cipriano, 2018). A Odontologia deve fazer parte dessa equipe, visto que a cavidade oral pode hospedar processos patológicos, bem como manifestar efeitos colaterais produzidos por medicamentos estabelecidos para a doença de base. (Oliveira \& Lima, 2019)

A presença do Cirurgião Dentista (CD) em ambiente hospitalar contribui para a prevenção de agravos e alterações orais de pacientes que se encontram em Unidades de Terapia Intensiva (UTI). A assistência odontológica no âmbito hospitalar tem se mostrado eficiente na incorporação de hábitos de higiene oral e redução do biofilme, determinação da condição bucal, realização do diagnóstico e tratamento de infecções odontogênicas e não odontogênicas, além de avaliar a necessidade de instalação de aparelhos bucais para prevenir ou tratar lesões (Rodrigues et al., 2019)

O tratamento de lesões bucais traumáticas pode ser realizado através da proservação ou pela associação entre a remoção do fator causal e do uso de medicamentos para redução da dor. Buscando amenizar os sintomas e acelerar o processo de cicatrização, para que haja um aumento na qualidade de vida desses pacientes, a terapia de fotobiomodulação pode ser 
incorporada como alternativa de tratamento como método não invasivo (Valle, Karam, Rezende, Zangrando, \& Damante,2017).

O Laser (Amplificação de Luz por Emissão Estimulada de Radiação) é uma fonte de luz que possui inúmeras indicações na odontologia. A terapia com o laser de baixa potência é conhecida também como Terapia de Fotobiomodulação. Essa terapêutica estimula efeitos biológicos e tem a capacidade de modular eventos metabólicos através de processos fotoquímicos e fotofísicos, aumentando o metabolismo celular pela estimulação da atividade mitocrondrial, resultando em modulação do processo inflamatório, efeito analgésico, antiedematoso e cicatrizador, bem como se observa a produção de colágeno e elastina, contração da ferida e aceleração da cicatrização (Reolon, Rigo, Conto \& Cé, 2017; Oliveira, Martins, Ribeiro \& Paula, 2019; Mansouri et al., 2020; Hendler et al., 2021; Lago, 2021). Estudos mostraram evidências favoráveis à TFBM em lesões bucais desde que seja em dose ideal e que esta terapia propicie tratamento e alívio dos sintomas (Valle et al., 2017; Santos et al., 2018; Oliveira et al., 2019; Luke, Mathew, Altawash \& Madan, 2019; Lago, 2021).

Este trabalho tem como objetivo relatar um caso de uso da terapia de fotobiomodulação com o laser de baixa potência no tratamento de lesões bucais em paciente com doença neurológica, por um cirurgião-dentista habilitado, em um hospital de alta complexidade em São Luís, Maranhão.

\section{Metodologia}

Partindo da permissão por meio do Termo de Consentimento Livre e Esclarecido (TCLE) em partilhar as imagens para devido fim, o presente estudo trata de um relato de caso clínico de cunho analítico descritivo (Pereira et al., 2018), realizado em um hospital de alta complexidade em São Luís-MA.

As informações contidas neste trabalho foram obtidas por meio de revisão do prontuário, entrevista com o acompanhante do paciente, registro fotográfico dos procedimentos realizados aos quais o paciente foi submetido e revisão da literatura.

\section{Relato de Caso}

Paciente M.J.C.B, sexo masculino, 43 anos, admitido em UTI de um hospital de alta complexidade em São Luís- MA, com história de queda de própria altura após crise convulsiva, pico hipertensivo e dispneia, onde foram evidenciados rebaixamento de nível de consciência (RNC), Glasgow 4+1t+3, taquicardia e HAS. Com diagnóstico prévio de neurosequela por AVC isquêmico há 4 anos e tromboembolismo pulmonar, sem outras comorbidades e sem história de tabagismo ou etilismo. Durante internação, evoluiu de tubo orotraqueal para traqueostomia, permanecendo em ventilação mecânica, apresentando hipertonia intensa e decorticação ao ser estimulado, realizando autofagia do lábio inferior como consequência da falta de coordenação dos movimentos musculares característicos da sequela neurológica e potencializados durante manipulação (troca de curativo ou aspiração traqueal). 
Figura 1: Aspecto da Lesão inicial.

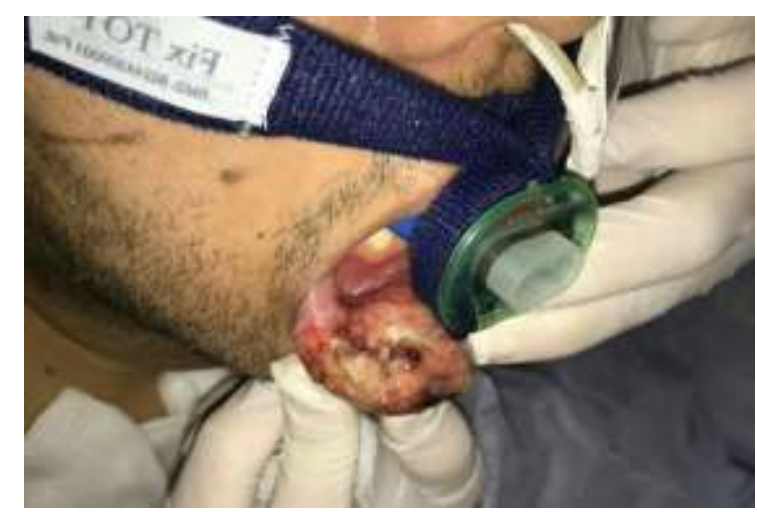

Fonte: Autores.

Ao exame físico odontológico, foi observado se tratar de um paciente com todos os dentes em bom estado, onde os elementos 11 e 12 estavam vestibularizados, havendo uma lesão ulcerada extensa com áreas de tecido de granulação em lábio inferior, estendendo-se para mucosa interna labial, de consistência endurecida e profundidade importante. O mesmo estava utilizando cânula de Guedel, como indicação da Fisioterapia, porém a mesma comprimia ainda mais os tecidos moles, além de causar lesões iatrogênicas no palato duro. Como terapia oferecida pela equipe médica, foi aplicada Triancinolona Acetonida que se mostrou sem efeito terapêutico.

Figura 2: (A) Confecção e instalação da placa oclusal; (B) Desgaste das faces incisais dos dentes 11 e 12.
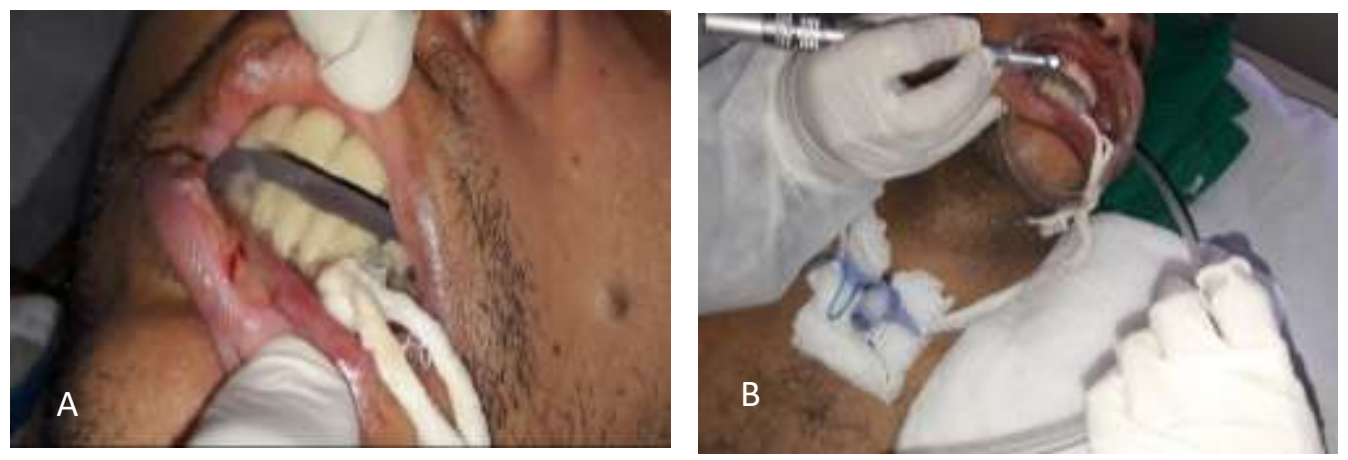

Fonte: Autores.

O plano terapêutico odontológico constituiu-se de remoção imediata da cânula de Guedel, otimização da analgesia, gerenciamento e orientação de higiene bucal à equipe de enfermagem, confecção de placa oclusal na tentativa de afastar tecidos moles, desgaste de faces incisais de dentes 11 e 12, além da suspensão do uso de Triancinolona Acetonida. A manutenção de cuidado odontológico intensivo (limpeza da ferida; limpeza da placa; e colocação da placa de forma intermitente) foi realizado por 07 (sete) dias e posteriormente indicada a aplicação de laser de baixa potência como terapia adjuvante. 
Figura 3: (A) e (B) Aspecto da lesão 07 (sete) dias após cuidados odontológicos intensivos; (C) Irradiação com o laser de baixa potência no comprimento de onda $660 \mathrm{~nm}$.
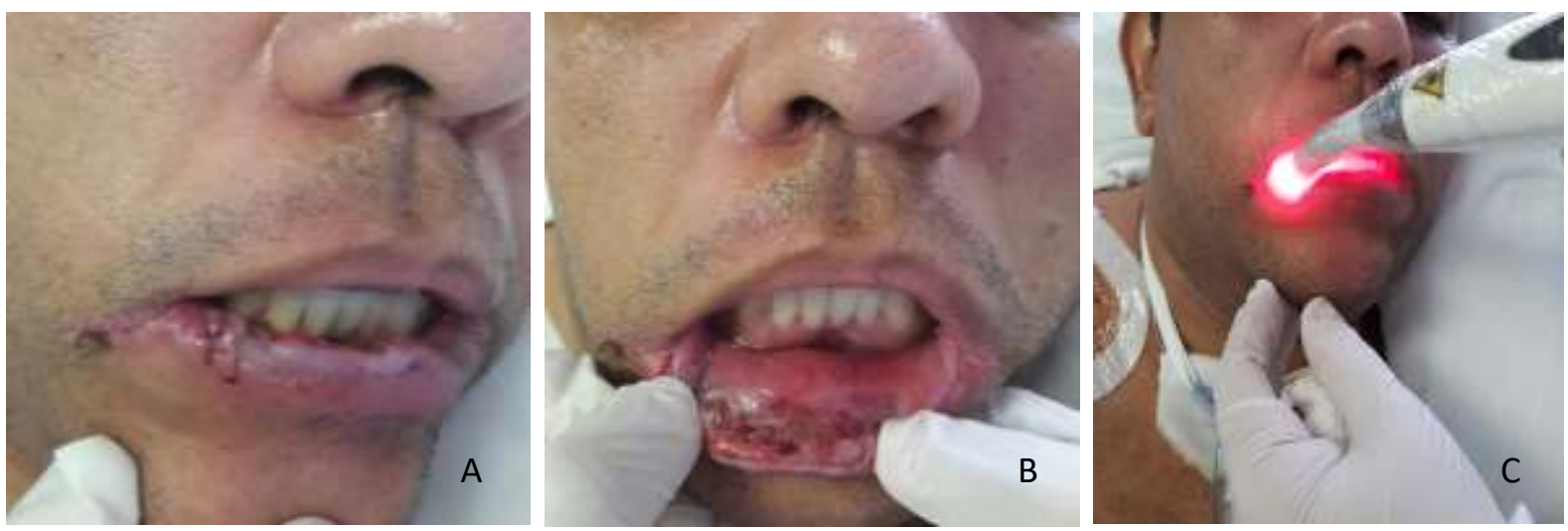

Fonte: Autores.

O equipamento utilizado na terapia de fotobiomodulação foi Laser Duo ${ }^{\circledR}$ da MMOptics. A irradiação foi realizada com o laser de baixa potência no comprimento de onda de $660 \mathrm{~nm}$, potência de $100 \mathrm{~mW}$, tempo 20 segundos, densidade de energia de $66 \mathrm{~J} / \mathrm{cm}^{2}$, energia $2 \mathrm{~J}$, área de spot de $0,03 \mathrm{~cm}^{2}$, com aplicação pontual em 9 pontos distribuídos na lesão, cobrindo toda a região em 3 sessões realizadas em dias consecutivos, sendo necessário o paciente estar sedado durante os procedimentos odontológicos.

Após a instituição do plano terapêutico e 24 horas após a aplicação da terapia de fotobiomodulação, houve expressiva reparação da lesão no lábio. Diminuição na extensão e profundidade da lesão, aspecto do tecido epitelial mais vascularizado, eritematoso, porém sem sinais flogísticos ou sangramento.

Figura 4: (A) e (B) Aspecto da lesão após cuidados odontológicos intensivos e as 3 (três) sessões de TFBM.
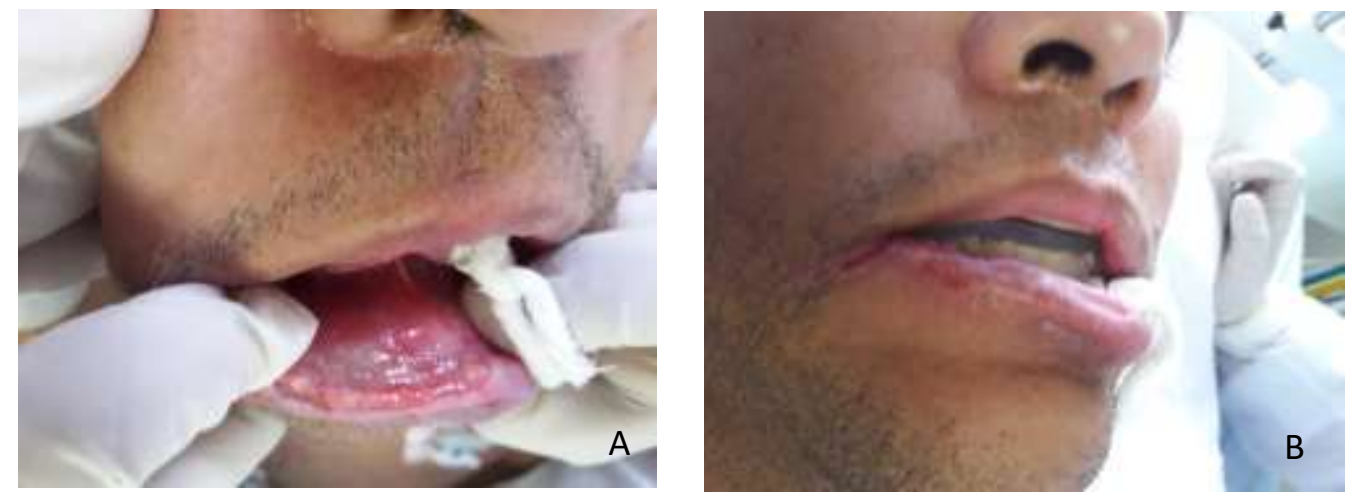

Fonte: Autores.

Seguindo o Protocolo Clínico para o tratamento de trauma em tecidos moles sugerido pelo fabricante, em três sessões seguidas e mantidos o protetor bucal, analgesia, orientação de higiene bucal aos cuidadores e comunicada a equipe de odontologia do setor de transferência, após 24 horas de desmame de ventilação mecânica o paciente recebeu alta da UTI para dar seguimento ao cuidado odontológico em enfermaria. 


\section{Discussão}

A terapia de fotobiomodulação tem sido utilizado em diversas áreas da Odontologia como método não invasivo, proporcionando alívio das dores durante e após intervenções odontológicas, ou como modalidade terapêutica no tratamento de lesões com condições dolorosas e inflamatórias. Tendo como finalidade o restabelecimento do equilíbrio biológico celular, melhorando as condições de vitalidade tecidual (Lins et al., 2010; Santos et al., 2018; Oliveira et al, 2019; Luke et al., 2019; Mansouri et al., 2020; Lago, 2021).

Neste relato de caso, o paciente apresentou lesão labial ulcerada extensa por trauma, com áreas de tecido de granulação em lábio inferior. O plano terapêutico proposto como método adjuvante aos cuidados odontológicos intensivos consistiu na aplicação da terapia de fotobiomodulação. A irradiação foi realizada com o laser vermelho, com comprimento de onda de $660 \mathrm{~nm}$, potência de $100 \mathrm{~mW}$, tempo 20 segundos, energia $2 \mathrm{~J}$ em toda a extensão da lesão. Após 24 horas da primeira aplicação, houve expressiva melhora na regressão da lesão, observando-se diminuição da profundidade, sugerindo que a TFBM pode ser uma opção de tratamento no auxílio da cicatrização de lesões bucais.

No caso relatado, a regressão da lesão e o aumento da cicatrização ocorreu possivelmente devido à ação dos efeitos do processo de reparação tecidual durante a terapia a laser: a proliferação celular, a síntese colagênica e o aumento da circulação local (Lins et al., 2010; Wagner et al., 2013; Luke et al., 2019; Hendler et al., 2021; Lago, 2021). Além disso os lasers de baixa potência são reconhecidos pela ação analgésica: estimulando a liberação de endorfinas, controlando os mediadores da dor e inibindo sinais nociceptores; a modulação do processo inflamatório e efeito antiedematoso: atuando na redução do edema e hiperemia vascular e efeito cicatrizante: acelerando a cicatrização dos tecidos, estimulando o reparo e remodelação óssea, restaurando a função neural e agindo na modulação de células do sistema imune para facilitar o processo de reparo (Lins et al., et al., 2010; Wagner et al., 2013; Reolon et al., 2017; Santos et al., 2018; Hendler et al., 2021; Lago, 2021).

Em revisão sistemática Khaleel Ahmed et al. (2020) compararam a terapia de fotobiomodulação com o uso medicamentos tópicos para o tratamento de úlceras bucais. Os medicamentos usados foram Triancinolona acetonida, Amlexanox, Granofurin e Solcoseryl. Os pacientes tratados com o laser relataram menor dor e diminuição das lesões, semelhante ao caso apresentado. A TFBM se mostrou mais eficiente em comparação aos medicamentos utilizados.

Wagner et al. (2013) verificaram em um estudo experimental em ratos que fototerapia acelerou a cicatrização de feridas nos animais, e as alterações mais expressivas ocorreram após 5 dias de irradiação. Os autores concluíram que a terapia promove uma re-epitelização mais organizada e mais rápida da mucosa bucal, fato esse que corrobora com nossos achados.

Valle et al. (2017) apresentaram relato de 3 casos diferentes com a aplicação da TFBM: úlcera traumática, reação inflamatória a corpo estranho e queilite angular de origem traumática, obtendo sucesso em sessão única, sendo eficaz na redução dos sintomas de forma imediata e na aceleração da cicatrização das lesões, esses resultados se assemelham ao caso clínico exposto.

O tratamento com o laser também se mostrou superior no estudo de Ribeiro e Marangoni (2020), quando utilizaram a fotobiomodulação em uma criança de 8 anos de idade com úlcera traumática. Na Escala Visual Analógica (EVA), que consiste em escore de aferição de dor pelo paciente, o paciente relatou escore de dor 8(oito). A úlcera não cicatrizava há mais de um ano, devido a Síndrome de Brodie, caracterizada pelo excesso de vestibularização dos molares superiores. O tratamento tópico empregado anteriormente ao uso do laser foi o Sulfato de Neomicina com Tartarato de Bismuto. Os resultados foram semelhantes aos achados neste trabalho. Após 24 horas da aplicação da TFBM, observou-se início de reparação tecidual e ausência de exsudato na região da mucosa jugal. Após 9 dias, houve total reparação da lesão e informação de diminuição considerável da dor relatada pelo paciente para escore 3 (três).

Sabe-se que a condição bucal e necessidade de tratamento em pacientes hospitalizados necessitam de acompanhamento do Cirurgião-dentista (CD) (Sousa et al., 2019; Macedo, Pereira, Corrêa, Pinho \& Casanovas, 2020). Os 
cuidados bucais quando realizados de forma adequada promovem ações de prevenção de lesões e seu tratamento precoce (Rodrigues et al., 2019; Macedo et al., 2020).

A abordagem odontológica em Cuidados Paliativos (CP) visa à manutenção da saúde oral e intervenção no alívio da dor, melhorando a qualidade de vida do paciente (Oliveira \& Lima, 2019). O uso da terapia de fotobiomodulação se mostra eficiente na aceleração da cicatrização de lesões bucais em paciente sob $\mathrm{CP}$, portanto a importância da utilização do laser pelo CD na unidade hospitalar possibilita a redução a prescrições medicamentosas, conforto e, por conseguinte, diminuição de custos de internação (Rodrigues et al., 2019; Oliveira et al., 2019). No nosso estudo, o paciente neurosequelado é dependente de cuidados e incapaz de realizar as atividades de vida diárias, os cuidados paliativos odontológicos foram implementados com o uso da TFBM como terapia não invasiva com excelentes resultados após a primeira aplicação, o que possibilitou a suspensão de terapia medicamentosa usada anteriormente sem sucesso.

\section{Conclusão}

Baseado nos achados deste caso, pode-se concluir que o tratamento de lesão bucal traumática em paciente com doença neurológica utilizando a TFBM, associado a um cuidado odontológico intensivo, tem demonstrado ser uma alternativa rápida e eficaz, com aceleração no processo de cicatrização e sem efeitos colaterais.

No entanto, ainda é considerada uma alternativa terapêutica recente e requer conhecimento técnico sobre sua utilização em diferentes áreas hospitalares. Sendo assim, mais estudos e relatórios clínicos de acompanhamento são necessários para assentar essa terapia de suporte para pacientes hospitalizados.

\section{Referências}

Cipriano, P. (2018). Cuidados Paliativos em Doentes com Acidente Vascular Cerebral: Um Estudo Retrospetivo de 5 Anos de uma Equipa Intra-Hospitalar de Suporte em Cuidados Paliativos. Medicina Interna, 25(3), 186-192. https://doi.org/10.24950/rspmi/original/288/3/2018

Gomes, S. I. F. (2019). A efetividade da intervenção do enfermeiro especialista em enfermagem de reabilitação na deglutição comprometida em doentes pós acidente vascular cerebral. (Dissertação de mestrado) Instituto Politécnico de Viseu; Viseu, Portugal.

Hendler, K. G., Canever, J. B., de Souza, L. G., das Neves, L. M. S., de Cássia Registro Fonseca, M., Kuriki, H. U., da Silva Aguiar Junior, A., Barbosa, R. I., \& Marcolino, A. M. (2021). Comparison of photobiomodulation in the treatment of skin injury with an open wound in mice. Lasers in Medical Science.

Khaleel Ahmed, M., Jafer, M., Nayeem, M., Hussain Moafa, I., Quadri, M. F. A., Gopalaiah, H., \& Quadri, M. F. A. (2020). Low-level laser therapy and topical medications for treating aphthous ulcers: A systematic review. Journal of Multidisciplinary Healthcare, 13, $1595-1605$.

Lago, A. D. N. (2021). Laser na odontologia [recurso eletrônico]: conceitos e aplicações clínicas. In EDUFMA (Vol. 1). https://doi.org/10.1128/AAC.0372814

Lins, R. D. A. U., Dantas, E. M., Lucena, K. C. R., Catão, M. H. C. V., Granville-Garcia, A. F., \& Neto, L. G. C. (2010). Efeitos bioestimulantes do laser de baixa potência no processo de reparo. An Bras Dermatol, 85(6), 849-855.

Luke, A. M., Mathew, S., Altawash, M. M., \& Madan, B. M. (2019). Lasers: A review with their applications in oral medicine. Journal of Lasers in Medical Sciences, 10(4), 324-329.

Macedo, M. M., Abreu-Pereira, C. A., Corrêa, N. de C., Pinho, J. R. O., \& Casanovas, R. C. (2020). Perfil Bucal De Pacientes Internados Em Uti Adulto. Rev. Rede Cuid. Saúde, 14(2), 29-42.

Mansouri, V., Arjmand, B., Tavirani, M. R., Razzaghi, M., Rostami-Nejad, M., \& Hamdieh, M. (2020). Evaluation of Efficacy of Low-Level Laser Therapy. Journal of Lasers in Medical Sciences, 11(4), 369-380.

Oliveira, C. S. de, Montenegro, C. P. D., \& Lima, A. M. da C. (2019). Odontologia e cuidados paliativos: estudo de caso. Rev. Longeviver, 4.

Oliveira, F. A. M. de., Martins, M. T., Ribeiro, M. A., Mota, P. H. A. da., \& Paula, M. V. Q. de. (2018). Indicações e tratamentos da laserterapia de baixa intensidade na odontologia: uma revisão sistemática da literatura TT - HU Rev, 44(1), 85-96.

Pereira, A. S., Shitsuka, D. M., Parreira, F. J., \& Shitsuka, R. (2018). Metodologia da pesquisa científica. Santa Maria, RS: UFSM, NTE.

Reolon, L. Z.,Rigo, L., Conto, F. de., \& Cé, L. C. (2017). Impacto da laserterapia na qualidade de vida de pacientes oncológicos portadores de mucosite oral. Rev Odontol UNESP, 46(1), 19-27. 
Research, Society and Development, v. 10, n. 6, e47110616083, 2021

(CC BY 4.0) | ISSN 2525-3409 | DOI: http://dx.doi.org/10.33448/rsd-v10i6.16083

Ribeiro, T. E., \& Marangoni, A. F. (2020). Avaliação do efeito da fotobiomodulação em úlcera traumática em crianças: relato de caso clínico. Revista Cientifica UMC, 5(1), 1-12.

Rodrigues, A. C. A., Parreira, M. G. de O., Santos, P. C. M., Magalhães, S. R., Melgaço, C. A., \& Jorge, K. O. (2019). Odontologia hospitalar: atuação do cirurgião-dentista na unidade de terapia intensiva. Revista de Ensino Pesquisa e Extensão, 20(1), 282-297.

Santos, L. B dos., \& Waters, C. (2020). Perfil epidemiológico dos pacientes acometidos por acidente vascular cerebral: revisão integrativa. Brazilian Journal of Development. 6(1): 2749-2775.

Santos, T. K. G. L. dos., Alcoforado, L. G. de C., Leitão, A. de S., Vanderlei, A. C. de Q. V., Carvalho, A. K. F. A., \& Vieira, A. P. de S. B. (2018). Uso da laserterapia de baixa potência no tratamento de lesões orais. R e v $i$ s t a $C$ a m $p$ o d o $S$ a b e R, 4, 240-257.

Sousa, J. P. D. L., Sousa, F. S. de, Souza, L. D., Corrêa, N. de C., Rodrigues, V. P., \& Casanovas, R. C. (2019). Levantamento das ações de cuidados bucais em unidades de terapia intensiva adulto no contexto multiprofissional. Rev Pesq Saúde, 20(2), 62-66.

Valle, L. A., Karam, P. S. B. H., Rezende, M. L. R. de., Zangrando, M. S. R., \& Damante, C. A. (2017). Laser de baixa intensidade no tratamento de úlceras traumáticas e queilite angular: relatos de casos. REV ASSOC PAUL CIR DENT, 71(1), 30-34.

Wagner, V. P., Meurer, L., Martins, M. A. T., Danilevicz, C. K., Magnusson, A. S., Marques, M. M., Filho, M. S., Squarize, C. H., \& Martins, M. D. (2013). Influence of different energy densities of laser phototherapy on oral wound healing. Journal of Biomedical Optics, 18(12), 128002. 